\title{
BMJ Open Impacts of COVID-19 and SARS-CoV-2 on male reproductive function: a systematic review and meta- analysis protocol
}

\author{
Xinyun Li (D) , ${ }^{1,2}$ Hua Lu (D) , ${ }^{1,2}$ Fangyuan Li, ${ }^{1,2}$ Qi Zhang, ${ }^{1,2}$ Tong Wang, ${ }^{1,2}$ \\ Lingxia Qiang, ${ }^{1,2}$ Qian Yang ${ }^{1,2}$
}

To cite: Li X, Lu H, Li F, et al. Impacts of COVID-19 and SARS-CoV-2 on male reproductive function: a systematic review and metaanalysis protocol. BMJ Open 2022;12:e053051. doi:10.1136/ bmjopen-2021-053051

- Prepublication history for this paper is available online. To view these files, please visit the journal online (http://dx.doi. org/10.1136/bmjopen-2021053051).

Received 02 May 2021 Accepted 31 October 2021
A) Check for updates

(C) Author(s) (or their employer(s)) 2022. Re-use permitted under CC BY-NC. No commercial re-use. See rights and permissions. Published by BMJ.

${ }^{1}$ Gynecology, Hospital of Chengdu University of Traditional Chinese Medicine, Chengdu, People's Republic of China ${ }^{2}$ School of Clinical Medicine, Chengdu University of Traditional Chinese Medicine, Chengdu, People's Republic of China

Correspondence to

Dr Hua Lu;

Ih18980880525@126.com

\section{ABSTRACT}

Introduction COVID-19 pandemic caused by SARSCoV-2 has become a global health challenge. SARS-CoV-2 can infect host cells via the ACE2 receptor, which is widely expressed in the corpus cavernosum, testis and male reproductive tract, and participates in erection, spermatogenesis and androgen metabolism. Also, the immune response and persistent fever resulting from COVID-19 may lead to damage of the testicular activity, consequently compromising male fertility.

Methods and analysis PubMed, MEDLINE, EMBASE, Web of Science, Cochrane Library, China National Knowledge Infrastructure, China Science and Technology Journal database, Chinese Biomedical Databases and Wanfang Data will be systematically searched for observational studies (case-control and cohort) published up to March 2021 in English or in Chinese literature on the impacts of COVID-19 and SARS-CoV-2 on male reproductive function. This protocol will follow the Preferred Reporting Items for Systematic Review and Meta-Analysis Protocols guidelines and Meta-analysis of Observational Studies in Epidemiology. The primary outcome will be semen parameters, and the additional outcomes will include: (a) detection of SARS-COV-2 in semen, (b) male sexual hormones, (c) sperm DNA fragmentation index, (d) erectile function, (e) evaluation of testis and also the male genital tract. Two reviewers will independently extract data from the included studies based on a predesigned data extraction form. The risk of bias of included studies will be evaluated through the Newcastle-Ottawa Scale for observational studies. Review Manager software V.5.3 will be used for statistical analysis. Q statistic and $I^{2}$ test will be performed to assess the heterogeneity among studies. Sensitivity analysis will be used to explore the robustness of pooled effects. We will use the Grading of Recommendations Assessment, Development and Evaluation system to assess the quality of evidence. Ethics and dissemination Ethical approval is not required and results will be published in a peer-reviewed journal.

PROSPERO registration number CRD42021245161.

\section{INTRODUCTION}

COVID-19, caused by SARS-CoV-2, is a relatively high infectious, rather heterogeneous
Strengths and limitations of this study

- This systematic review will summarise and evaluate the observational studies about the impacts of COVID-19 and SARS-CoV-2 on male reproductive function with multidimensional perspectives, to update the understanding of COVID-19 and SARS-CoV-2.

- The review will be conducted under the Preferred Reporting Items for Systematic Reviews and MetaAnalyses guidelines, with regular consensus meetings and a third reviewer to reduce reviewer bias.

- Fertility is the vital health issue for all of humanity, and we will provide evidence to support the development of standard treatment strategies and management measures.

- There have been other systematic reviews of relevant topics, and the heterogeneity of the studies as well as the small number of studies may still be potential limitation, and it will be better to have a longer follow-up for male reproductive assessment.

- Potential biases from the original studies in observational studies may be potentially misleading.

and relatively long incubation period of disease. ${ }^{12}$ And the continued outbreak of COVID-19 pandemic has become a global health challenge quickly. As of April 2021, more than 129 million confirmed infections have been reported worldwide, and infection cases ranged from asymptomatic to critical illness which can affect different even multiple organ systems involved with variable symptoms. ${ }^{3}$ Compared with women, men seem to be vulnerable in this public health crisis. A recent study, through literature search and analysis, found that men are more susceptible to being infected with SARS-CoV-2 than women. ${ }^{4}$ In addition, a scoping review and meta-study collected 61 studies from 11 countries (59 254 patients), and epidemiological results showed that male patients had a higher mortality rate. ${ }^{5}$ However, the high 
fever, cytokine storm and virus invasion may also have adverse effects on the male reproductive system, which is raising concern as to whether SARS-CoV-2 infection can directly or potentially threatens the male fertility and causes the pathophysiological changes of male reproductive system or not.

As a novel Betacoronavirus, SARS-CoV-2 is an enveloped, positive sense, single stranded RNA virus. It contains four important structural proteins namely nucleocapsid proteins $(\mathrm{N})$, envelope proteins $(\mathrm{E})$, membrane proteins (M) and spike proteins (S). Together these proteins complete the assembly and infection of the virus. In the process of interaction between SARS-CoV-2 and host cell, $\mathrm{S}$ protein is cleaved into two subunits by host protease. The $\mathrm{S} 1$ subunit of $\mathrm{S}$ protein recognises and binds to ACE2 receptor on the surface of host cell, while S2 subunit facilitates membrane fusion of virus and host cell, and finally enters host cell through endocytosis. ${ }^{67}$ In addition, transmembrane protease serine protease 2 (TMPRSS2) on cell membrane is also involved in the cleavage of S protein, which activate ACE2-mediated virus invasion. ${ }^{7}$ The whole genome sequence of the SARSCoV-2 maintains $\sim 80 \%$ nucleotide identity to the original SARS epidemic viruses, ${ }^{8}$ and SARS-CoV-2 also uses human ACE2 as its receptor. However, structural changes in SARS-CoV-2 receptor-binding motif result in higher hACE2-binding affinity than that of SARS-CoV, which also leads to be more infectious and aggressive. ${ }^{910}$

Cell entry receptors are the key to the tropism and severity of virus infection of a specific virus. ${ }^{11}$ ACE2, which is the major 'gate' for SARS-CoV-2 to invade host cells, is actually not expressed much in the lung by analysing different databases of human transcriptome and experimental models, mainly in the small parts of type II alveolar epithelial cells. ${ }^{12-14}$ The respiratory symptoms of COVID-19 may be considered the release and infiltration of inflammatory cytokines caused by SARS-CoV-2, and the increase of ACE2 expression. ${ }^{15}{ }^{16}$ On the contrary, small intestine, testis, kidney, myocardium, colon and thyroid are the overlapping tissues with the highest level of ACE2 expression, ${ }^{12}$ which also reveals that SARS-CoV-2 infection is actually a multi-system disease.

Thus, the extensive expression of ACE2 and TMPRSS2 in corpus cavernosum, testis and male reproductive tract may imply a potential risk with SARS-CoV-2 invasion on male reproductive system, such as erectile function, spermatogenesis, sperm motility and androgen metabolism. Indeed, it was found that SARS-CoV-2 can be detected not only in semen samples of patients with severe clinical conditions and high viral load, but also during the acute or the recovering phase of infection. ${ }^{1718}$ Currently, limited understanding of the impact of COVID-19 and SARSCoV-2 on the reproductive system is perhaps one of the biggest challenges. And it is still unclear whether it would have any long-term effects on male fertility following recovery from SARS-CoV-2 infection, and whether the infection of SARS-CoV-2 on gonadal cells would have a possible short-term and long-term impact on gonocyte differentiation during early stage of spermatogenesis in early pubertal and pre-pubertal children. ${ }^{19}$ There is an urgent need to provide more information to inform key decisions, such as whether testicular function monitoring is required regularly, whether sperm is a potential vector of SARS-CoV-2? Even though the risk of SARS-CoV-2 shedding into the semen may be minor, it is also unacceptable for infertile couples. ${ }^{4}$ And whether the infected male patients need to avoid unprotected sexual relations, assisted reproductive technology, gamete donation and so on. And standard treatment strategies should be established in time to help male patients recovering from COVID-19.

This review protocol aims to identify the impacts of COVID-19 and SARS-CoV-2 on male reproductive function, and whether semen is a way of sexual transmission for the virus.

\section{METHODS AND ANALYSIS}

The proposed systematic review and meta-analysis is consistent with the Preferred Reporting Items for Systematic Review and Meta-Analysis Protocols guidelines ${ }^{20}$ and Meta-analysis of Observational Studies in Epidemiology, ${ }^{21}$ and the protocol has been registered in the international Prospective Register of Systematic Review.

\section{Eligibility criteria}

All observational designs (case-control and cohort) which evaluated the reproductive function of male who confirmed infection with SARS-CoV-2 will be included. There will be no restrictions concerning ethnicity, publication status, geography or medical conditions. All publications in English and Chinese will be included. Semen parameters, such as ejaculate volume, total number, sperm concentration, sperm motility, sperm vitality, sperm morphology, will be the primary outcome. Additional outcomes will consist of (a) detection of SARSCOV-2 in semen, (b) male sexual hormones, (c) sperm DNA fragmentation index, (d) erectile function, (e) evaluation of testis and also the male genital tract. Articles with no access to the full text or which are not available in English or Chinese language will be excluded from the study. Studies with insufficient or incomplete data will also be excluded.

\section{Information sources}

Two independent reviewers will systematically search the following databases: PubMed, MEDLINE, EMBASE, Web of Science, Cochrane Library, China National Knowledge Infrastructure, China Science and Technology Journal database, Chinese Biomedical Databases and Wanfang Data, identifying all related articles up to March 2021 in English or in Chinese literature on the impacts of COVID-19 and SARS-CoV-2 on male reproductive function. Grey literature will be searched in OpenGrey. Consensus meetings will be held at each stage; in case of disagreement, the third reviewer will take part in the 


\begin{tabular}{|c|c|}
\hline No & Search items \\
\hline 1 & SARS-CoV-2 \\
\hline 2 & Severe Acute Respiratory Syndrome coronavirus 2 \\
\hline 3 & COVID-19 \\
\hline 4 & Coronavirus Disease 2019 \\
\hline 5 & 2019-nCOV \\
\hline 6 & 2019 Novel Coronavirus \\
\hline 7 & OR/1-6 \\
\hline 8 & Male reproductive function \\
\hline 9 & Male reproducti ${ }^{\star}$ \\
\hline 10 & Male reproductive health \\
\hline 11 & Male reproductive system \\
\hline 12 & Male gonadal function \\
\hline 13 & Male infertil ${ }^{*}$ \\
\hline 14 & Male fertil ${ }^{*}$ \\
\hline 15 & Semen* \\
\hline 16 & Sperm* \\
\hline 17 & Male genital ${ }^{*}$ \\
\hline 18 & OR/8-17 \\
\hline 19 & Observational study \\
\hline 20 & Cohort study \\
\hline 21 & Case-control study \\
\hline 22 & OR/19-21 \\
\hline 23 & 7 AND 18 AND 22 \\
\hline
\end{tabular}

solution. The reference lists of included studies will be searched manually for other relevant publications. And all the searches will be re-run before the final analysis to ensure data integrity and consistency.

\section{Search strategy}

Table 1 shows the search strategy for PubMed, and the reviewers will adapt it to other databases.

\section{Study selection}

All the articles retrieved from the databases will be imported into EndNote V.X9. Two reviewers will independently screen the titles and abstracts, and remove the duplicates and non-conforming studies based on the eligibility criteria. The final decision of included studies will be made after reading the full text. A third reviewer will examine the included and excluded studies and identify the reasons for each decision. Consensus meetings will be held at each stage; disagreements will be settled through two reviewers, or consult the third reviewer for arbitration. Figure 1 presents the Preferred Reporting Items for Systematic Reviews and MetaAnalyses flowchart to summarise the entire process of the study selection.

\section{Data extraction and management}

Two reviewers will independently extract data from the included studies based on a predesigned data extraction form. The data extraction form includes the following items: first author, year of publication, study location (country), study design, sample size, participant characteristics (age, race, duration and severity of SARS-CoV-2 infection, thus recovered, asymptomatic, mild, moderate or severe/critical cases, diagnostic method, semen sample collection method, tested tissues, with or without fever, with or without other comorbid conditions), interventions, follow-up and primary and secondary results of all included studies. Consensus meetings will be held at each stage; disagreement will be resolved by consultation with the third reviewer. All data will be cross-checked before statistical analysis using the Review Manager software (V.5.3).

\section{Risk of bias in individual studies}

Methodologic quality assessment of included studies will be made for potential risk of bias with the use of the Newcastle-Ottawa scale (NOS) for observational studies. ${ }^{22}$ NOS consists of eight items divided into three perspectives: the selection of the study groups; the comparability of the groups and exposure or outcome of interest for case-control or cohort studies, respectively. Two reviewers will independently assess the risk of bias, give each study a score of 0-9, with 9 being the maximum score, and produce a table that specified those risks. Discrepancies will be discussed to reach an agreement. If necessary, a third review author will be consulted. A final score $>6$ will be regarded as high quality.

\section{Analysis}

All statistical analyses will be performed using the Review Manager software (V.5.3), and a $\mathrm{p}$ value $<0.05$ will be considered statistically significant. Dichotomous data will be analysed by using the risk ratio or ORs with $95 \%$ CIs. $^{23}$ Continuous outcomes data measured on the same scale will be expressed as a mean value and SD and be analysed by using weighted mean differences. $\chi^{2}$ test statistic (Q) and $\mathrm{I}^{2}$ test will be performed to assess the heterogeneity among studies. Q statistic will be conducted to find the presence of heterogeneity, and $\mathrm{I}^{2}$ test will be conducted to calculated percentage of variation due to heterogeneity. If the $Q$ value is $>0.05$, it indicates that outcome variable is statistically significant. If $\mathrm{I}^{2}$ value is $<50 \%$ and $\mathrm{p}$ value is $>0.1$, the fixed effect model will be chosen. If $\mathrm{I}^{2}$ is $\geq 50 \%$ or $\mathrm{p}$ is $<0.1$, the random effect model will be chosen. And if the heterogeneity is substantial, the results will not be combined. When substantial heterogeneity occurs, we will conduct subgroup analysis and meta-regression to explore the source of heterogeneity. If considerable heterogeneity cannot be reduced by some methods, or the source of heterogeneity cannot be explored by subgroup analysis or meta-regression, we will conduct a systematic review with narrative analysis and tabulate the results. If the heterogeneity of the data is acceptable, we 


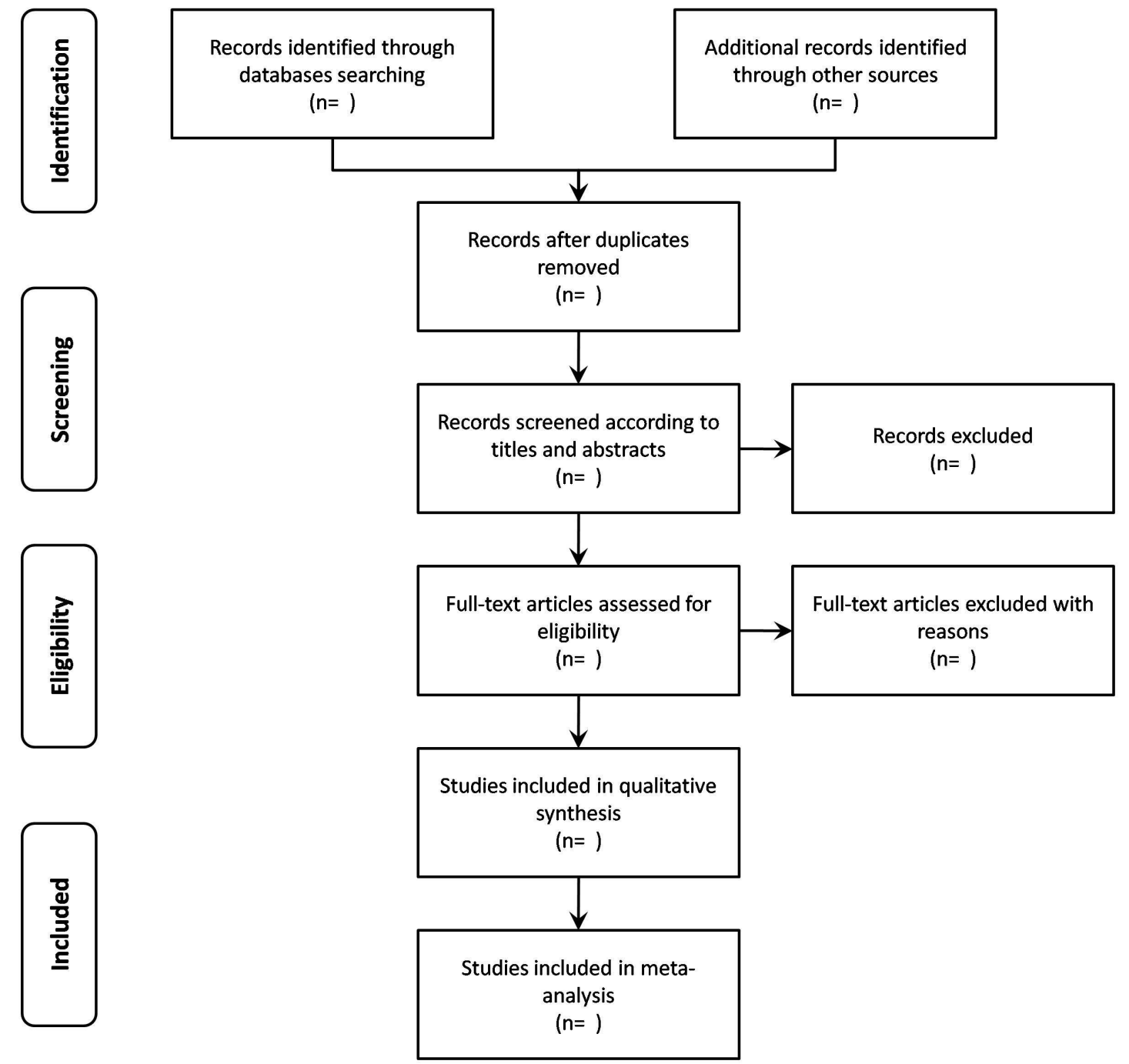

Figure 1 PRISMA flowchart of the study selection. PRISMA, Preferred Reporting Items for Systematic Reviews and MetaAnalyses.

will continue to conduct a meta-analysis. Sensitivity analysis will be used to explore the robustness of the pooled effects of the included studies regarding the study quality and sample size. Publication bias will be assessed by using a funnel plot, Egger's test and Begg's test.

\section{Confidence in cumulative evidence}

We will use the Grading of Recommendations Assessment, Development and Evaluation system to assess the quality of evidence as very low, low, moderate and high quality. Two reviewers will carry out this assessment independently, and any differences will be decided through discussion or consultation with the third reviewer.

\section{Patient and public involvement}

No patient will be involved.

\section{Ethics and dissemination}

As patients or private data will not be collected, there are no ethical considerations and no need for ethical approval. The results will be published in a peer-reviewed journal.

\section{DISCUSSION}

In continuous outbreaks of COVID-19, one of the most important concerns in reproductive health problems is the reproductive function of infected male patients. Studies reported that ACE2 and TMPRSS2 are expressed in the testis and male genital tract, and regulated by testosterone, which indicate that male genitalia is susceptible to infection by SARS-CoV-2. Mature spermatozoa even have all the mechanisms needed to bind to SARSCoV-2. ${ }^{24}$ Hajizadeh Maleki et al evaluated multiple seminal biomarkers and reproductive function among male patients with laboratory-confirmed COVID-19 and healthy controls, which could provide direct experimental evidence of targeting and damaging the male reproductive system by SARS-CoV-2 infection. ${ }^{26}$ And sperm parameters of the contaminated samples post-warming may be shifted in assisted reproductive technology, since the survival of the virus at cooling temperature is not excluded. ${ }^{27}$ Although precise mechanisms of male reproductive damages are still unclear, persistent high temperature and secondary autoimmune inflammation may also 
be the blows to male reproductive system. ${ }^{28}$ COVID-19 and SARS-CoV-2 have been reported to have direct and indirect negative effects on male reproductive function, ${ }^{29-31}$ but, there is an urgent need for further secondary analysis to confirm the impact of COVID-19 and SARS-CoV-2 on male reproductive function, and develop standard treatment strategies to help male patients recover from the attack of COVID-19 pandemic.

Contributors $\mathrm{XL}$ and $\mathrm{HL}$ conceptualised and designed the protocol. The manuscript of the protocol was drafted by $X \mathrm{~L}$ and critically revised by $\mathrm{HL}$ and $\mathrm{FL}$. The search strategy was developed by XL and FL. LQ and QY will conduct the study selection. QZ and TW will be in charge of data extraction, and statistical analysis will be performed by XL, FL and QZ. HL will be the third party and host consensus meetings at each stage in case of disagreement. All authors read, revised and approved the final manuscript.

Funding This study was funded by the Ministry of Science and Technology of the People's Republic of China (grant number: 2018YFC1704305).

Competing interests None declared.

Patient and public involvement Patients and/or the public were not involved in the design, or conduct, or reporting, or dissemination plans of this research.

Patient consent for publication Not applicable.

Provenance and peer review Not commissioned; externally peer reviewed.

Open access This is an open access article distributed in accordance with the Creative Commons Attribution Non Commercial (CC BY-NC 4.0) license, which permits others to distribute, remix, adapt, build upon this work non-commercially, and license their derivative works on different terms, provided the original work is properly cited, appropriate credit is given, any changes made indicated, and the use is non-commercial. See: http://creativecommons.org/licenses/by-nc/4.0/.

\section{ORCID iDs}

Xinyun Li http://orcid.org/0000-0002-6739-5735

Hua Lu http://orcid.org/0000-0002-2709-222X

\section{REFERENCES}

1 Wang C, Wang Z, Wang G, et al. COVID-19 in early 2021: current status and looking forward. Signal Transduct Target Ther 2021;6:114

2 Grasselli G, Zangrillo A, Zanella A, et al. Baseline characteristics and outcomes of 1591 patients infected with SARS-CoV-2 admitted to ICUs of the Lombardy region, Italy. JAMA 2020;323:1574-81.

3 Gupta A, Madhavan MV, Sehgal K, et al. Extrapulmonary manifestations of COVID-19. Nat Med 2020;26:1017-32.

$4 \mathrm{He} \mathrm{W}$, Liu X, Feng L, et al. Impact of SARS-CoV-2 on male reproductive health: a review of the literature on male reproductive involvement in COVID-19. Front Med 2020;7:594364.

5 Borges do Nascimento IJ, Cacic N, Abdulazeem HM, et al. Novel coronavirus infection (COVID-19) in humans: a scoping review and meta-analysis. J Clin Med 2020;9:941.

6 Satarker S, Nampoothiri M. Structural proteins in severe acute respiratory syndrome Coronavirus-2. Arch Med Res 2020;51:482-91.

$7 \mathrm{Hu} \mathrm{B}$, Guo H, Zhou P, et al. Characteristics of SARS-CoV-2 and COVID-19. Nat Rev Microbiol 2021;19:141-54.

8 Gralinski LE, Menachery VD. Return of the coronavirus: 2019-nCoV. Viruses 2020;12:135.

9 Shang J, Ye G, Shi K, et al. Structural basis of receptor recognition by SARS-CoV-2. Nature 2020;581:221-4.
10 Chen Y, Guo Y, Pan Y, et al. Structure analysis of the receptor binding of 2019-nCoV. Biochem Biophys Res Commun 2020;525:135-40.

11 Scialo F, Daniele A, Amato F, et al. Ace2: the major cell entry receptor for SARS-CoV-2. Lung 2020;198:867-77.

12 Wang Y, Wang Y, Luo W, et al. A comprehensive investigation of the MRNA and protein level of ACE2, the putative receptor of SARS-CoV-2, in human tissues and blood cells. Int J Med Sci 2020;17:1522-31.

13 Zou X, Chen K, Zou J, et al. Single-cell RNA-seq data analysis on the receptor ACE2 expression reveals the potential risk of different human organs vulnerable to $2019-n C o V$ infection. Front Med 2020;14:185-92.

14 Serfozo P, Wysocki J, Gulua G, et al. Ang II (Angiotensin II) Conversion to Angiotensin-(1-7) in the Circulation Is POP (Prolyloligopeptidase)-Dependent and ACE2 (Angiotensin-Converting Enzyme 2)-Independent. Hypertension 2020;75:173-82.

15 Zhuang M-W, Cheng Y, Zhang J, et al. Increasing host cellular receptor-angiotensin-converting enzyme 2 expression by coronavirus may facilitate 2019-nCoV (or SARS-CoV-2) infection. J Med Virol 2020;92:2693-701.

16 Ziegler CGK, Allon SJ, Nyquist SK, et al. SARS-CoV-2 receptor ACE2 is an interferon-stimulated gene in human airway epithelial cells and is detected in specific cell subsets across tissues. Cell 2020;181:1016-35.

17 Saylam B, Uguz M, Yarpuzlu M, et al. The presence of SARS-CoV-2 virus in semen samples of patients with COVID-19 pneumonia. Andrologia 2021;53:e14145.

18 Li D, Jin M, Bao P, et al. Clinical characteristics and results of semen tests among men with coronavirus disease 2019. JAMA Netw Open 2020;3:e208292.

19 Anifandis G, Tempest HG, Oliva R, et al. COVID-19 and human reproduction: a pandemic that packs a serious punch. Syst Biol Reprod Med 2021;67:3-23.

20 Shamseer L, Moher D, Clarke M, et al. Preferred reporting items for systematic review and meta-analysis protocols (PRISMA-P) 2015: elaboration and explanation. BMJ 2015;350:g7647.

21 Stroup DF, Berlin JA, Morton SC, et al. Meta-Analysis of observational studies in epidemiology: a proposal for reporting. meta-analysis of observational studies in epidemiology (moose) group. JAMA 2000;283:2008-12.

22 Wells GA, Shea B, O'Connell D. The Newcastle- Ottawa scale (NOS) for assessing the quality of nonrandomised studies in meta-analyses. Ottawa:The Ottawa Hospital Research Institute. Available: http:// www.ohri.ca/programs/clinical_epidemiology/oxford.asp

23 Delgado-Rodríguez M, Sillero-Arenas M. Systematic review and meta-analysis. Med Intensiva 2018;42:444-53.

24 Pozzilli P, Lenzi A. Commentary: testosterone, a key hormone in the context of COVID-19 pandemic. Metabolism 2020;108:154252.

25 Paoli D, Pallotti F, Turriziani O, et al. SARS-CoV-2 presence in seminal fluid: myth or reality. Andrology 2021;9:23-6.

26 Hajizadeh Maleki B, Tartibian B. COVID-19 and male reproductive function: a prospective, longitudinal cohort study. Reproduction 2021;161:319-31.

27 Anifandis G, Messini Cl, Simopoulou M, et al. SARS-CoV-2 vs. human gametes, embryos and cryopreservation. Syst Biol Reprod Med 2021;67:260-9.

28 Youssef K, Abdelhak K. Male genital damage in COVID-19 patients: are available data relevant? Asian J Urol 2020;21.

29 Omolaoye TS, Adeniji AA, Cardona Maya WD, et al. SARS-COV-2 (Covid-19) and male fertility: where are we? Reproductive Toxicology 2021;99:65-70.

30 Sheikhzadeh Hesari F, Hosseinzadeh SS, AsI Monadi Sardroud MA. Review of COVID-19 and male genital tract. Andrologia 2021;53:e13914.

31 Haghpanah A, Masjedi F, Alborzi S, et al. Potential mechanisms of SARS-CoV-2 action on male gonadal function and fertility: current status and future prospects. Andrologia 2021;53:e13883. 